\title{
A Characterization of Approximation Resistance for Even $k$-Partite CSPs
}

\author{
Per Austrin* \\ Aalto University and KTH Royal Institute of Technology \\ Subhash Khot ${ }^{\dagger}$ \\ New York University and University of Chicago
}

\begin{abstract}
A constraint satisfaction problem (CSP) is said to be approximation resistant if it is hard to approximate better than the trivial algorithm which picks a uniformly random assignment. Assuming the Unique Games Conjecture, we give a characterization of approximation resistance for $k$-partite CSPs defined by an even predicate.
\end{abstract}

*Work done while at the University of Toronto supported by NSERC.

${ }^{\dagger}$ Research supported by NSF Expeditions grant CCF-0832795, NSF Waterman Award and BSF grant 2008059. 


\section{Introduction}

In the past 20 years, there has been a significant amount of work done on understanding the approximability of various constraint satisfaction problems (CSPs).

For the purposes of this paper, a CSP is defined by a $k$-ary predicate $P:\{-1,1\}^{k} \rightarrow\{0,1\}$ over a Boolean alphabet 1 An instance consists of a set of constraints, each of which dictates that $P$ applied to some list of $k$ literals should be satisfied (a literal is a variable or the negation of a variable). The objective is to find an assignment to the variables so as to maximize the number of satisfied constraints. Two well-known examples are MAx $k$-SAT (where $P$ is the disjunction of the $k$ input bits) and Max $k$-Lin (where $P$ is the parity of the $k$ input bits).

Essentially every MAX CSP is NP-hard (the exception being when $P$ only depends on one of its input bits). In terms of approximability, it is easy to see that choosing a uniformly random assignment to the variables, without even looking at the instance, yields an approximation ratio of $\left|P^{-1}(1)\right| / 2^{k}$, where $\left|P^{-1}(1)\right|$ is the number of inputs in $\{-1,1\}^{k}$ that satisfy $P$.

Improving upon this trivial algorithm turns out to be surprisingly difficult. In a groundbreaking paper, Goemans and Williamson GW95] used semidefinite programming (SDP) to give improved approximation algorithms for MAX 2-SAT and MAX 2-LIN. SDP was soon used to give better approximation algorithms for many other problems as well, but for some CSPs, perhaps most prominently MAX 3-SAT and MAX 3-Lin, no improvement over the random assignment algorithm was found. Then, in a new breakthrough, Håstad [Hås01] showed that such an improvement would not be possible: approximating MAX 3-SAT within 7/8 $+\epsilon$ or MAX 3-LiN within $1 / 2+\epsilon$ for some $\epsilon>0$ is NP-hard. In other words, MAX 3-SAT and MAX 3-Lin have the remarkable property that the completely mindless random assignment algorithm is optimal!

CSPs which have this property - that they are NP-hard to approximate within $\left|P^{-1}(1)\right| / 2^{k}+\epsilon$ - are called approximation resistant. Following Håstad's initial result, many more CSPs have been shown to be approximation resistant [GLST98, ST00, EH08, Has05. Fairly quickly, a complete characterization of approximation resistance for predicates of arity three was found: $P:\{-1,1\}^{3} \rightarrow$ $\{0,1\}$ is approximation resistant if and only if $P$ accepts all inputs of odd parity, or if it accepts all inputs of even parity Hås01, Zwi98.

However, the next small case, predicates of arity 4, is still not completely classified, and it is not at all clear whether there is a nice, clean characterization. We would like to emphasize that by a characterization we mean a necessary and sufficient condition. Modulo symmetries, there are 400 non-constant predicates of arity 4. Hast [Has05] showed 275 of these to be approximable, 79 of them to be approximation resistant, and left the status of the remaining 46 open.

In recent years, progress has been made on our understanding of approximation resistance under the assumption of the Unique Games Conjecture (UGC) [Kho02]. The first author and Mossel AM09] proved that assuming the UGC, $P$ is approximation resistant if there exists an unbiased pairwise independent distribution over $\{-1,1\}^{k}$ supported on $P^{-1}(1)$. Using this condition, it can be shown that as $k \rightarrow \infty$, an overwhelming fraction of all predicates are in fact approximation resistant AH11. A somewhat more (complicated and) general sufficient condition is known [AH12]. As in AM09, this condition is in terms of the biases and pairwise correlations of distributions supported on $P$. At this point, it seems unlikely that there is a clean characterization (necessary and sufficient), but one can hope that approximation resistance is at least decidable.

Relevant here is the work of Raghavendra Rag08, which shows assuming the UGC that for any CSP, its approximability threshold is determined by the integrality gap of a natural SDP relaxation

\footnotetext{
${ }^{1}$ As is common, the input bits are written in the $\{-1,1\}$ notation with -1 interpreted as logical True and 1 as logical False. Also "parity" corresponds to taking product of the bits: odd parity means the product is -1 and even parity means the product is 1 .
} 
for the problem. Furthermore, Raghavendra and Steurer [RS09] show that this integrality gap can be approximated to within an additive error $\epsilon$ (in time doubly exponential in $\epsilon$ ).

This "almost" shows that it is decidable to determine whether a CSP is approximation resistant. However, as we have no a priori bound on the error $\epsilon$ needed, it only shows that it is recursively enumerable to determine whether a CSP is approximable. Note that, for every $k$ there is a smallest gap $\epsilon_{k}$ such that any approximable predicate $P$ on $k$ bits can be approximated within at least $\left|P^{-1}(1)\right| / 2^{k}+\epsilon_{k}$. If this number $\epsilon_{k}$ can be computed, approximation resistance would be decidable, but it is possible (though seemingly unlikely) that $\epsilon_{k}$ tends to 0 faster than any computable function.

\subsection{Our Contribution}

The strength of Rag08, namely that it works in a black-box fashion for any CSP, is in some sense a weakness in this setting, as it is not explicit and does not give any insight into what structural properties cause a predicate to be approximation resistant. In this paper, we make progress towards an explicit characterization of approximation resistance. We restrict the class of CSPs we study in two ways.

1. We only consider $k$-partite instances. In a $k$-partite instance, the variables are grouped into $k$ layers, and in each constraint, the literal passed as the $i$ 'th argument to $P$ comes from the $i$ 'th layer.

2. We only consider $P$ which are even. $P$ is even if $P(x)=P(-x)$ for every $x \in\{-1,1\}^{k}$, where $-x$ denotes bitwise negation of $x$.

We refer to this as the MAx $\operatorname{PantCSP}(P)$ problem. Our main contribution is an explicit necessary and sufficient characterization (assuming the UGC) of when MAX $\operatorname{PARTCSP}(P)$ is approximation resistant. As in the case of [AM09] and its generalizations, our condition is based on the existence of certain distributions $\mu$ over the set of satisfying assignments of $P$ and furthermore the conditions on these distributions depend only on their pairwise correlations $\mathbb{E}_{\mu}\left[x_{i} x_{j}\right]$.

In order to properly state the characterization, we need to make a few definitions.

Definition 1.1. Let $G=(S, E)$ be a multigraph with vertex set $S \subseteq[k]$ and no self-loops. For a correlation matrix $\rho \in \mathbb{R}^{k \times k}$ we define $\rho(G)=\prod_{i j \in E} \rho_{i j}$. For a distribution $\Lambda$ over $k \times k$ correlation matrices we define $\Lambda(G)=\mathbb{E}_{\rho \in \Lambda}[\rho(G)]$.

The key part of our definition is the existence of distributions $\Lambda$ over correlation matrices each of which arises from a distribution over $P^{-1}(1)$ - we refer to these as $P$-supported correlation matrices - such that $\Lambda(G)$ vanishes on certain graphs. Specifically:

Definition 1.2. Let $\Lambda$ be a distribution over $k \times k$ correlation matrices. We say that $\Lambda$ is $m$ vanishing on $P$ if:

1. $\Lambda$ is a distribution over $P$-supported correlation matrices.

2. For every $S \subseteq[k]$ such that $\hat{P}(S) \neq 0$, and every odd-degree multigraph $G$ on $S$ with at most $m$ edges, it holds that $\Lambda(G)=0$.

Here $\hat{P}(S)$ denotes the Fourier coefficient of the predicate $P$ on the set $S$ (i.e. the coefficient of the monomial $\prod_{i \in S} x_{i}$ when $P$ is written as a multi-linear polynomial). Now we can state our main result.

Theorem 1.3. Assuming the $U G C, \operatorname{MAx} \operatorname{PARTCSP}(P)$ is approximation resistant if and only if for every positive integer $m$ there exists a distribution $\Lambda$ which is $m$-vanishing on $P$. 
Note that if there is a pairwise independent distribution supported on $P$, i.e., if the identity matrix is $P$-supported, then taking $\Lambda$ to be the singleton distribution on the identity matrix is $m$-vanishing on $P$ for every $m$. As such, this characterization generalizes the sufficient condition of [AM09].

Given $m$ and $P$, it is fairly easy to prove that the existence of an $m$-vanishing $\Lambda$ on $P$ is decidable. Hence the condition of Theorem 1.3 is recursively enumerable. We feel that this characterization is promising with respect to decidability. For instance, it is quite possible that one can show some explicit upper bound on the largest value of $m$ that one needs to check, which would immediately give decidability. We also remark that, even though we do not prove it here, the characterization in Theorem 1.3 is equivalent to saying that there is a distribution $\Lambda$ which is $m$-vanishing on $P$ for all $m$ simultaneously.

\subsection{Proof Ideas}

We now briefly and informally outline the main ideas of the proof of Theorem 1.3 .

Algorithm. Suppose there is no $m$-vanishing distribution $\Lambda$ for some $m$. By LP duality, there are then constants $\left\{\gamma_{G}\right\}$ such that $\sum_{G} \gamma_{G} \rho(G)>\delta$ for all $P$-supported $\rho$, where the sum is over all odd-degree $G$ on at most $m$ edges. Now, assume we are given a solution to the basic SDP relaxation for MAX PARTCSP $(P)$ with value 1 (in reality it will only have value close to 1 but this is just a small technicality). Then for each constraint we have a local distribution $\mu$ and since the SDP value is 1 its correlation matrix $\rho$ is $P$-supported. The basic idea is, very loosely, to design a rounding algorithm which, given some graph $G$, finds an assignment with value $\left|P^{-1}(1)\right| / 2^{k}+\rho(G)$. Picking a random $G$ with probability proportional to $\left|\gamma_{G}\right|$ then gives an assignment with value $\left|P^{-1}(1)\right| / 2^{k}+\Omega(\delta)$.

To get an assignment with value $\left|P^{-1}(1)\right| / 2^{k}+\rho(G)$, the idea is to do as follows. For simplicity, suppose $V(G)=[k]$ and consider the monomial $\prod_{i=1}^{k} x_{i}$. We can construct the solution iteratively edge by edge, as follows. Initially, set all $x_{i}=1$ (corresponding to the empty graph). Then, for an edge $e=(i, j)$, pick a standard Gaussian vector $\underline{\mathrm{g}}_{e}$, and multiply $x_{i}$ (resp. $\left.x_{j}\right)$ by $\left\langle\underline{\mathrm{g}}_{e}, \underline{\mathrm{v}}_{i}\right\rangle$ (resp. $\left\langle\underline{\mathrm{g}}_{e}, \underline{\mathrm{v}}_{j}\right\rangle$ ), where $\underline{\mathrm{v}}_{i}$ and $\underline{\mathrm{v}}_{j}$ are the vectors in the SDP solution corresponding to $x_{i}$ and $x_{j}$. This operation has the effect of multiplying $\mathbb{E}\left[\prod_{i \in[k]} x_{i}\right]$ by a factor $\left\langle\underline{\mathrm{v}}_{i}, \underline{\mathrm{v}}_{j}\right\rangle=\rho_{i j}$ where $\rho_{i j}$ is the correlation between $i$ and $j$ in the local distribution on $x_{1}, \ldots, x_{k}$. Repeating this for all edges of the graph, we get $\mathbb{E}\left[\prod_{i \in[k]} x_{i}\right]=\rho(G)$, and we can make sure that all other non-constant monomials have expectation 0 , meaning that we get an advantage of $\rho(G)$ over $\left|P^{-1}(1)\right| / 2^{k}+\rho(G)$.

To wrap this up and get the formal proof, there are som additional technicalities to account for: the values assigned by the above rounding are not Boolean-valued, we need to deal with negated literals, and we need to take the magnitude of the Fourier coefficients $\hat{P}(S)$ of $P$ into consideration. The formalization of the "monomial rounding" described above is given in Lemma 4.1 in Section 4.1 and its use to give a non-trivial algorithm for MAx $\operatorname{PARTCSP}(P)$ is then described in Section 4.3 ,

Hardness. As is by now standard, the task of proving hardness boils down to constructing a dictatorship test using the predicate $P$. The dictatorship test gets oracle access to $k$ functions $f_{1}, \ldots, f_{k}:\{-1,1\}^{n} \rightarrow\{-1,1\}$, and the question is whether $f_{1}=f_{2}=\ldots=f_{k}$ are all equal to some dictatorship function. The test operates by picking $k$ inputs $x_{1}, \ldots, x_{k}$ and then accepts iff $P\left(f_{1}\left(x_{1}\right), f_{2}\left(x_{2}\right), \ldots, f_{k}\left(x_{k}\right)\right)=1$. The restriction to only using $P$ as the acceptance predicate is what gives us hardness for $\operatorname{MAx} \operatorname{CSP}(P)$ rather than an arbitrary CSP, and the restriction that 
we have $k$ different functions and make one query to each, instead of a single function, is precisely what gives us hardness for Max PartCSP $(P)$ instead of MAx $\operatorname{CSP}(P)$.

Such a test is completely specified by the distribution of $\left(x_{1}, \ldots, x_{k}\right)$. To specify this we choose some very large $m$ and use the $m$-vanishing distribution $\Lambda$ guaranteed to exist. To sample $\left(x_{1}, \ldots, x_{k}\right)$, we do as follows: first sample a $P$-supported correlation matrix $\rho$ according to $\Lambda$, and let $\mu$ be some $P$-supported distribution with correlation matrix $\rho$. Then, for each $i \in[n]$ we sample the $i$ 'th coordinate $\left(x_{1}^{i}, \ldots, x_{k}^{i}\right)$ independently from $\mu$. The completeness of the test follows by $\mu$ being $P$-supported. The soundness follows using the invariance principle: first, we show that if the functions $f_{1}, \ldots, f_{k}$ have low influence the acceptance probability (appropriately arithmetized) can be well approximated by a multilinear polynomial in Gaussian variables with the same second moments as $x$. Since higher moments of Gaussian variables are determined by their covariance matrix, this multilinear polynomial (and therefore also the acceptance probability) can be expressed as a function of the covariance matrix, i.e., $\rho$, and it turns out that all terms except for the constant $\left|P^{-1}(1)\right| / 2^{k}$ are of the form $\rho(G)$ for some odd-degree graph $G$ on less than $m$ edges. Hence taking the expectation over $\rho \sim \Lambda$, all non-constant terms vanish.

Source of the various restrictions. It may be instructive to point out where the various restrictions we impose come into play.

$k$-partiteness. The fact that we know for each variable what "role" it will play is critical in allowing us to obtain the algorithm. In particular, in the "monomial rounding" described above, it is important that any given variable corresponds to some given vertex of the graph $G$ that we are using (the vertices of $G$ correspond to layers of the CSP instance). If a vertex could appear as several different vertices of $G$ (i.e., in several different layers), it is not clear how to round it in such a way that the different occurences don't interfere with each other.

Even predicates. This restriction is in some sense minor and more technical in nature. It allows us to assume that the distributions $\mu$ supported on $P^{-1}(1)$ are unbiased, which simplies may arguments. That said, it is not clear exactly how to generalize the present characterization to a general $P$.

Odd-degree graphs. The reason why the characterization only involves odd-degree graphs is essentially the presence of negated literals. First, in the algorithm it turns out that it is necessary for the graphs to be odd-degree, as this essentially ensures that we don't have cancellations when dealing with negated literals. Second, in the hardness result it turns out that it is sufficient for the graphs to have odd-degree, because the functions $f_{i}$ we are testing can be assumed to be odd by the standard technique of folding, which is implemented by introducing negated literals.

\subsection{Discussion}

On the unnecessity of pairwise independence. It is known that there are approximation resistant predicates which do not support a pairwise independent distribution. A basic such example is the predicate $G L S T:\{-1,1\}^{4} \rightarrow\{0,1\}$ defined by

$$
\operatorname{GLST}\left(x_{1}, x_{2}, x_{3}, x_{4}\right)=\left\{\begin{array}{ll}
x_{2} \neq x_{3} & \text { if } x_{1}=-1 \\
x_{2} \neq x_{4} & \text { if } x_{1}=1
\end{array} .\right.
$$

This predicate was shown to be approximation resistant by Guruswami et al. [GLST98, but there is no pairwise independent distribution supported on its accepting assignments - indeed it is not 
difficult to check that $x_{2} x_{3}+x_{2} x_{4}+x_{3} x_{4}<0$ for all accepting inputs. In [AH12], Theorem VIII.6, a generalization of the pairwise independence condition was given which also covers the GLST predicate and in fact as far as we are aware cover all currently known examples of approximation resistant predicates.

The condition of Theorem 1.3 essentially generalizes the condition of [AH12]. We say "essentially" because Theorem VIII.6 of [AH12 in some cases allows for a condition referred to as $\{i, j\}$-negativity, and it is not clear that this condition is captured by Theorem 1.3 . The only example given in [AH12] using the $\{i, j\}$-negativity condition is not an even predicate, so it is possible that this is a distinction between even $P$ and general $P$. On the other hand, it appears that for the example given in [AH12, one can prove approximation resistance without using $\{i, j\}$-negativity so it is not completely clear whether allowing this adds any new predicates. Another possibility is that this is a distinction between $\operatorname{Max} \operatorname{CSP}(P)$ and $\operatorname{Max} \operatorname{PartCSP}(P)$, because the proof in AH12 that $\{i, j\}$-negativity suffices does not extend to partite instances. In short, the situation is a bit of a mystery and may warrant further study.

On Max PartCSP $(P)$ vis-à-vis Max $\operatorname{CSP}(P)$. It is not known whether MAx PARTCSP $(P)$ behaves differently from MAx $\operatorname{CSP}(P)$ with respect to approximation resistance. Almost all proofs of approximation resistance for MAx $\operatorname{CSP}(P)$, including NP-hardness results such as [Hås01, EH08, can be adjusted to produce $k$-partite instances, thereby showing approximation resistance for $\operatorname{Max} \operatorname{PARTCSP}(P)$.

However, one exception is the result of Raghavendra Rag08, where it is not at all clear how to achieve this. If it were the case that the reduction of Rag08 can be adjusted to produce partite instances, our restriction to $k$-partite instances would have been without loss of generality (assuming the UGC), but as matters stand, this can not be deduced.

Another exception is the hardness derived in [AH12] from the $\{i, j\}$-negativity condition mentioned above.

\subsection{Outline}

In Section 2 we introduce notation and terminology used throughout the paper and state some known theorems that we need. In Section 3 we describe how to decide whether an $m$-vanishing distribution exists. We then proceed to prove Theorem 1.3, giving an algorithm in Section 4 and proving hardness in Section 5 ,

\section{Notation and definitions}

As is common, for convenience of notation we use $\{-1,1\}$ for Boolean values rather than $\{0,1\}$. Throughout, $P$ denotes a $k$-ary predicate $P:\{-1,1\}^{k} \rightarrow\{0,1\}$ which we assume to be even, i.e., $P(x)=P(-x)$ for all $x$.

We say a distribution $\mu$ over $\{-1,1\}^{k}$ is $P$-supported if $\operatorname{supp}(\mu) \subseteq P^{-1}(1)$. Similarly a correlation matrix $\rho \in \mathbb{R}^{k \times k}$ is $P$-supported if there is a $P$-supported $\mu$ such that $\rho_{i j}=\mathbb{E}_{\mu}\left[x_{i} x_{j}\right]$ for all $i, j$. Note that since $P$ is even, any $P$-supported distribution can without loss of generality be assumed to be unbiased, i.e., satisfying $\mathbb{E}_{\mu}\left[x_{i}\right]=0$ for all $i$, as far as its correlation matrix is concerned (since we can spread the probability mass equally on any pair of assignments $x$ and $-x$ without affecting the correlation matrix).

For the purposes of this paper, a multigraph is a graph $G$ which may have multiple edges but no self-loops. A multigraph has odd degree if every vertex of the graph has odd degree (when edges are 
counted with multiplicities). A key role in our characterization is played by multigraphs $G$ whose vertex set is some subset $S \subseteq[k]$, we refer to this as a multigraph on $S$.

We write $S^{n}$ for the $n$-dimensional unit sphere (i.e., the set of unit vectors in $\mathbb{R}^{n+1}$, and for two vectors $\underline{\mathrm{u}}, \underline{\mathrm{v}} \in \mathbb{R}^{n}$ we write $\langle\underline{\mathrm{u}}, \underline{\mathrm{v}}\rangle$ for their standard inner product.

\subsection{Partite Max-CSP and its SDP relaxation}

An instance $\Psi$ of MAx PARTCSP $(P)$ has $k \cdot n$ Boolean variables indexed by $[k] \times[n]$. Each constraint is of the form $P\left(b_{1} x_{1, j_{1}}, b_{2} x_{2, j_{2}}, \ldots, b_{k} x_{k, j_{k}}\right)$ for some indices $j_{1}, \ldots, j_{k}$ and some signs $b_{1}, \ldots, b_{k} \in\{-1,1\}$.

We use the following notation. The constraints of an instance are $\left(T_{1}, P_{1}\right),\left(T_{2}, P_{2}\right), \ldots$, where $T_{i} \subseteq[k] \times[n]$ are the set of variables that the $i$ 'th constraint depends on - exactly one from each layer - and $P_{i}:\{-1,1\}^{T_{i}} \rightarrow\{0,1\}$ is $P$ applied to the variables of $T_{i}$, possibly with some variables negated.

We say that $\Psi$ is $\alpha$-satisfiable if there is an assignment to the variables which satisfies an $\alpha$ fraction of all the constraints.

The basic SDP relaxation is described in Figure 1. It has as variables a vector $\underline{\mathrm{v}}_{i, j} \in S^{n \cdot k}$ for every variable $x_{i, j}$, and an unbiased distribution $\mu_{i}$ over $\{-1,1\}^{T_{i}}$ for each constraint $\left(T_{i}, P_{i}\right)$. The fact that this is a relaxation follows from the following observation: for any global integral assignment $\sigma \in\{-1,1\}^{k \cdot n}$, let $\mathcal{D}$ be the uniform distribution over the pair of integral assignments $\sigma$ and $-\sigma$. Let $\mu_{i}$ be the restrictions of $\mathcal{D}$ to the respective sets $T_{i}$ and $\mathbf{v}_{i, j}=\sigma_{i, j}$ be a 1-dimensional vector. Then it is easy to see that this is a feasible solution to the SDP and its objective is same as the fraction of constraints satisfied by $\sigma$ (or $-\sigma)$. Here we use the evenness of the predicate.

\section{Maximize $\sum_{i} \underset{x \sim \mu_{i}}{\mathbb{E}}\left[P_{i}(x)\right]$}

Subject to $\mu_{i}$ is an unbiased distribution over $\{-1,1\}^{T_{i}}$ for every $i$

$$
\begin{array}{ll}
\underline{\mathrm{v}}_{i, j} \in S^{n \cdot k} & \text { for all }(i, j) \in[k] \times[n] \\
\left.\mu_{i}\right|_{T}=\left.\mu_{j}\right|_{T} & \text { where } T=T_{i} \cap T_{j} \\
\left\langle\underline{\mathrm{v}}_{i_{1}, j_{1}}, \underline{\mathrm{v}}_{i_{2}, j_{2}}\right\rangle=\underset{x \sim \mu_{l}}{\mathbb{E}}\left[x_{i_{1}, j_{1}} x_{i_{2}, j_{2}}\right] & \text { for all }\left(i_{1}, j_{1}\right),\left(i_{2}, j_{2}\right) \in T_{l}
\end{array}
$$

Fig. 1: SDP relaxation of $\operatorname{MAx} \operatorname{PARTCSP}(P)$.

\subsection{The Unique Games Conjecture}

In this section, we state the formulation of the Unique Games Conjecture that we will use.

Definition 2.1. An instance $\Lambda=(U, V, E, \Pi,[L])$ of Unique Games consists of an unweighted bipartite multigraph $G=(U \cup V, E)$, a set $\Pi$ of constraints, and a set $[L]$ of labels. For each edge $e \in E$ there is a constraint $\pi_{e} \in \Pi$, which is a permutation on $[L]$. The goal is to find a labeling $\ell: U \cup V \rightarrow[L]$ of the vertices such that as many edges as possible are satisfied, where an edge $e=(u, v)$ is said to be satisfied by $\ell$ if $\ell(v)=\pi_{e}(\ell(u))$. 
Definition 2.2. Given a Unique Game instance $\Lambda=(U, V, E, \Pi,[L])$, let $\operatorname{Opt}(\Lambda)$ denote the maximum fraction of simultaneously satisfied edges of $\Lambda$ by any labeling, i. e.,

$$
\operatorname{Opt}(\Lambda):=\frac{1}{|E|} \max _{\ell: U \cup V \rightarrow[L]} \mid\{e: \ell \text { satisfies } e\} \mid .
$$

Conjecture 2.3. ([Kho02]) For every $\gamma>0$, there is an integer $L$ such that, for Unique Games instances $\Lambda$ with label set $[L]$ it is NP-hard to distinguish between

- $\operatorname{Opt}(\Lambda) \geq 1-\gamma$

- $\operatorname{Opt}(\Lambda) \leq \gamma$.

\subsection{Analytic Tools}

Any Boolean function $f:\{-1,1\}^{n} \rightarrow \mathbb{R}$ can be written uniquely as a multilinear polynomial

$$
f(x)=\sum_{T \subseteq[n]} \hat{f}(T) \chi_{T}(x)
$$

where $\hat{f}(T)$ are the Fourier coefficients of $f$ and $\chi_{T}(x)=\prod_{i \in T} x_{i}$. As such, $f$ can be viewed as a multilinear polynomial $f: \mathbb{R}^{n} \rightarrow \mathbb{R}$ and this is the view we commonly take. We write $f \leq d$ for the part of $f$ that is of degree $\leq d$, i.e., $f^{\leq d}(x)=\sum_{|S| \leq d} \hat{f}(S) \chi_{S}(x)$.

Fact 2.4.

$$
\mathbb{E}[f(X)]=\hat{f}(\emptyset) \quad \operatorname{Var}[f(X)]=\sum_{T \neq \emptyset} \hat{f}(T)^{2}
$$

where the expectations are over a uniform $X$ in $\{-1,1\}^{n}$.

Definition 2.5. The influence of the $i$ 'th variable on $f$ is

$$
\operatorname{Inf}_{i}(f)=\sum_{T \ni i} \hat{f}(T)^{2}
$$

and the low-degree influence is

$$
\operatorname{Inf}_{i}^{\leq d}(f)=\operatorname{Inf}_{i}(f \leq d)=\sum_{\substack{T \ni i \\|T| \leq d}} \hat{f}(T)^{2} .
$$

As is common, the main analytic tool in our hardness result is the invariance principle [MOO10, Mos10. In particular, we have the following theorem.

Theorem 2.6. For every $k, \epsilon>0$ there is a $\delta>0$ such that the following holds.

Let $\mu$ be an unbiased distribution over $\{-1,1\}^{k}$ with $\min _{x \in\{-1,1\}^{k}} \mu(x) \geq \epsilon, X$ be a random $k \times n$ matrix over $\{-1,1\}$ with each column distributed according to $\mu$, independently, and $G$ be a random $k \times n$ matrix of standard Gaussians with the same covariance structure as $X$.

Then for any $k$ multilinear polynomials $f_{1}, \ldots, f_{k}: \mathbb{R}^{n} \rightarrow \mathbb{R}$ with $\operatorname{Inf}_{j}^{\leq 1 / \delta}\left(f_{i}\right) \leq \delta$ and $\operatorname{Var}\left[f_{i}\right] \leq 1$ for all $i \in[k], j \in[n]$, we have

$$
\left|\underset{X}{\mathbb{E}}\left[\prod_{i=1}^{k} f_{i}\left(X_{i}\right)\right]-\underset{G}{\mathbb{E}}\left[\prod_{i=1}^{k} f_{i}^{\leq 1 / \delta}\left(G_{i}\right)\right]\right| \leq \epsilon .
$$


Theorem 2.6 can be derived using Theorem 4.2 and Lemma 6.2 of [Mos10]: using Lemma 6.2 it follows that $\mathbb{E}_{X}\left[\prod_{i=1}^{k} f_{i}\left(X_{i}\right)\right]$ is close to $\mathbb{E}_{X}\left[\prod_{i=1}^{k} f_{i}^{\leq 1 / \delta}\left(X_{i}\right)\right]$ for sufficiently small $\delta$. Then we can use Theorem 4.2 on the functions $\left\{f_{i}^{\leq 1 / \delta}\right\}$. In Theorem 4.2, the values of $f_{i}^{\leq 1 / \delta}\left(X_{i}\right)$ and $f_{i}^{\leq 1 / \delta}\left(G_{i}\right)$ are truncated to the range $[0,1]$. By scaling this holds with $[0,1]$ replaced by some interval $[-B, B]$. It is not too hard to show that for sufficiently large $B$ (as a function of $k$ and $\epsilon$ ) truncation to the interval $[-B, B]$ does not change $\mathbb{E}\left[\prod_{i=1}^{k} f_{i}^{\leq 1 / \delta}\left(X_{i}\right)\right]$ (resp. $\mathbb{E}\left[\prod_{i=1}^{k} f_{i}^{\leq 1 / \delta}\left(G_{i}\right)\right]$ ) by more than $\epsilon$.

\subsection{Products of Gaussians}

We need the following Lemma about the expectation of a product of gaussians in terms of their pairwise correlations.

Lemma 2.7. Let $r$ be an integer and let $g_{1}, \ldots, g_{r}$ be gaussians with mean 0 , variance 1 , and covariance matrix $\rho$. Then

$$
\mathbb{E}\left[\prod_{i=1}^{r} g_{i}\right]=\sum_{M} \prod_{i j \in M} \rho_{i j},
$$

where $M$ ranges over all perfect matchings of the complete graph on $r$ vertices (if $r$ is even there are $(r-1)$ !! terms and if $r$ is odd the expectation is 0$)$.

\section{Decidability of $m$-vanishing Distributions}

Proposition 3.1. Given $P$ and $m$, the existence of a $\Lambda$ which is $m$-vanishing on $P$ is decidable.

Proof. There are $M \leq 2^{k} \cdot k^{2 m}$ graphs $G_{1}, \ldots, G_{M}$ of interest namely graphs with at most $m$ edges supported on some vertex set $S \subseteq[k]$. We need to decide whether there is a distribution $\Lambda$ on $P$-supported correlation matrices such that

$$
\underset{\rho \in \Lambda}{\mathbb{E}}\left[\left(\rho\left(G_{1}\right), \ldots, \rho\left(G_{M}\right)\right)\right]=\mathbf{0} .
$$

By Carathéodory's theorem this implies that $\Lambda$ can be assumed to have support at most $M$. Each $\rho$ in the support of $\Lambda$ can be represented using $\left|P^{-1}(1)\right|+k^{2}+1$ real variables, representing a distribution $\mu$ over $P^{-1}(1)$, its correlation matrix $\rho$, and finally its probability under $\Lambda$. The constraints that each $\rho$ is the correlation matrix of the corresponding $\mu$ and that $\Lambda\left(G_{i}\right)=0$ for every $G_{i}$ can be written as a finite number of polynomial equations in the $M\left(\left|P^{-1}(1)\right|+k^{2}+1\right)$ variables 2 In other words the set of $m$-vanishing $\Lambda$ of support size $\leq M$ form an algebraic set, so determining whether such $\Lambda$ exists boils down to determining whether this algebraic set is nonempty, which is decidable Tar51].

\section{Algorithm}

In this section we give an approximation algorithm with approximation ratio strictly larger than $\left|P^{-1}(1)\right| / 2^{k}$ for predicates $P$ which do not satisfy the condition of Theorem 1.3 . Thus, there exists an $m$ such that for every distribution $\Lambda$ (over $P$-supported correlation matrices), there is an odddegree multigraph $G$ over $S \subseteq[k]$ with at most $m$ edges such that $\hat{P}(S) \neq 0$ and $\Lambda(G) \neq 0$. For the rest of this section, fix this value of $m$.

\footnotetext{
${ }^{2}$ The variables representing probabilities need to be non-negative. This can be effected by taking them to be squares of respective variables.
} 


\subsection{Rounding Monomials}

First, we give an algorithm which will allow us to "pick up" a contribution proportional to $\rho(G)$ for any monomial, where $\rho$ is the correlation matrix of the local distribution (given by the SDP) on that monomial and $G$ is any graph. Lemma 4.1 below formalizes the high-level idea given in Section 1.2. Recall that the variables of the CSP are partitioned into $k$ layers, there are $n$ variables in each layer, and the SDP relaxation is as in Figure 1.

Lemma 4.1. Let $S \subseteq[k]$ be a set of layers, $G$ be an odd-degree multigraph on $S$, and $\tau>0$. Then for all sufficiently large $B \geq$ poly $(\log 1 / \tau)$ (where the polynomial depends only on $G$ ) there is a polynomial time algorithm which, given an SDP solution as in Figure 1 outputs an assignment $\alpha: S \times[n] \rightarrow[-1,1]$ to the layers of $S$ such that the following holds.

Let $V: S \rightarrow[n]$ be any choice of variables, one from each layer in $S$. Then

$$
\mathbb{E}\left[\prod_{i \in S} \alpha_{i, V(i)}\right]=\frac{\rho(G)}{B^{|S|}} \pm \tau,
$$

where $\rho$ is the correlation matrix defined by the SDP solution on these $k$ variables, i.e., $\rho_{i_{1}, i_{2}}=$ $\left\langle\underline{v}_{i_{1}, V\left(i_{1}\right)}, \underline{v}_{i_{2}, V\left(i_{2}\right)}\right\rangle$.

Proof. The algorithm works as follows. For each edge $e \in E(G)$, pick a standard Gaussian vector $\underline{\mathrm{g}}_{e}$, independently. For a vertex $u \in V(G)$, let $E(u) \subseteq E(G)$ denote the set of edges incident on $u$. For a variable $x_{i, j}$ such that $i \in S$, set

$$
\beta_{i, j}=\prod_{e \in E(i)}\left\langle\underline{\mathrm{g}}_{e}, \underline{\mathrm{v}}_{i, j}\right\rangle .
$$

Then, set

$$
\alpha_{i, j}= \begin{cases}\frac{\beta_{i, j}}{B} & \text { if }\left|\beta_{i, j}\right| \leq B \\ 0 & \text { otherwise }\end{cases}
$$

Fix $V: S \rightarrow[n]$ as in the statement. Now let us analyze $\mathbb{E}\left[\prod_{i \in S} \alpha_{i, V(i)}\right]$. Note that without the truncation when $\left|\beta_{i, V(i)}\right|$ exceeds $B$, the expectation would be exactly equal to (where in the second step below we use the independence of the Gaussians to move the expectation inside the product)

$$
\begin{aligned}
\mathbb{E}\left[\frac{1}{B^{|S|}} \prod_{i \in S} \beta_{i, V(i)}\right] & =\frac{1}{B^{|S|}} \mathbb{E}\left[\prod_{i \in S} \prod_{e \in E(i)}\left\langle\underline{\mathrm{g}}_{e}, \underline{\mathrm{v}}_{i, V(i)}\right\rangle\right] \\
& =\frac{1}{B^{|S|}} \prod_{(a, b)=e \in E(G)} \mathbb{E}\left[\left\langle\underline{\mathrm{g}}_{e}, \underline{\mathrm{v}}_{a, V(a)}\right\rangle\left\langle\underline{\mathrm{g}}_{e}, \underline{\mathrm{v}}_{b, V(b)}\right\rangle\right] \\
& =\frac{1}{B^{|S|}} \prod_{(a, b) \in E(G)}\left\langle\underline{\mathrm{v}}_{a, V(a)}, \underline{\mathrm{v}}_{b, V(b)}\right\rangle=\frac{\rho(G)}{B^{|S|}}
\end{aligned}
$$

Thus we want to bound the expectation of $\left|\prod_{i \in S} \frac{\beta_{i, V(i)}}{B}-\prod_{i \in S} \alpha_{i, V(i)}\right|$ by $\tau$. This can be shown to be of order $m \cdot \exp \left(-B^{2 / m} / 2\right)$, because each $\beta_{i, V(i)}$ is a product of at most $|E(G)| \leq m$ independent gaussians. Thus setting $B$ of order $(\log 1 / \tau)^{m / 2}$ we get the desired error bound. 


\subsection{Setup for the Algorithm}

Let $M \leq 2^{k} \cdot k^{2 m}$ be the number of odd-degree graphs on some $S \subseteq[k]$ with $\hat{P}(S) \neq 0$ and at most $m$ edges, and let $G_{1}, \ldots, G_{M}$ be these graphs. Further write $S_{t}=V\left(G_{t}\right) \subseteq[k]$ for the vertex set of $G_{t}$. For a correlation matrix $\rho \in \mathbb{R}^{k \times k}$, let $q(\rho)$ be the vector $q(\rho)=\left(\rho\left(G_{1}\right), \rho\left(G_{2}\right), \ldots, \rho\left(G_{M}\right)\right) \in \mathbb{R}^{M}$, and let $Q \subseteq \mathbb{R}^{M}$ be the convex hull of $\{q(\rho): \rho$ is $P$-supported $\}$.

Note that a $\Lambda$ such that $\Lambda\left(G_{i}\right)=0$ for all $1 \leq i \leq M$ is precisely a convex combination $\Lambda$ of $\rho$ 's such that $\mathbb{E}_{\rho \sim \Lambda}[q(\rho)]=\underline{0}$. In other words since $P$ does not satisfy the condition of Theorem 1.3 we have that $Q$ does not contain the origin. Furthermore $Q$ is compact and so we can find a separating hyperplane $\left(\gamma_{1}, \ldots, \gamma_{M}\right)$ such that $\sum_{t=1}^{M} \gamma_{t} \rho\left(G_{t}\right)>\delta$ for every $P$-supported $\rho$ and some universal constant $\delta$ (depending only on $P$ ).

Now let $\tau=\frac{\delta}{4 M}$ and set $B=\operatorname{poly}(\log 1 / \tau)$ large enough to make Lemma 4.1 work for all the graphs $G_{1}, \ldots, G_{M}$. In our algorithm, we are going to choose one $t \in[M]$ at random and then the algorithm is going to focus solely on the terms involving layers $S_{t}$. More precisely, as we shall see

in the next section, $t$ should be chosen with probability proportional to $\left|\frac{\gamma_{t}}{\hat{P}\left(S_{t}\right)}\right| \cdot B^{\left|S_{t}\right|}$. In order for this to make sense, we therefore need that

$$
\sum_{t=1}^{M}\left|\frac{\gamma_{t}}{\hat{P}\left(S_{t}\right)}\right| \cdot B^{\left|S_{t}\right|} \leq 1 .
$$

Fortunately, we can assume without loss of generality that this holds: since $B^{\left|S_{t}\right|} \leq B^{k}$ depends sub-linearly (in fact even poly-logarithmically) on $1 / \tau$, dividing each $\gamma_{t}$ by some factor $f>1$ causes $\delta$ and $\tau$ to also be divided by $f$, which in turn changes $B^{\left|S_{t}\right|}$ to poly $\log (f / \tau)=o\left(f B^{\left|S_{t}\right|}\right)$, so that the sum in the left hand side of (11) decreases by a factor which is super-constant in $f$. Hence choosing $f$ a sufficiently large constant, we can make (1) hold.

\subsection{The Rounding Algorithm}

We are now ready to describe the algorithm. Without loss of generality, we may assume that we are given a $(1-\epsilon)$-satisfiable $\operatorname{MAx} \operatorname{PARTCSP}(P)$ instance where $\epsilon>0$ is some sufficiently small constant (depending on $P$ ) to be determined later. If the instance is not $(1-\epsilon)$-satisfiable then a random assignment already gives an approximation ratio of $\frac{\left|P^{-1}(1)\right|}{2^{k}(1-\epsilon)}$.

By Markov's inequality, for at least a $1-\sqrt{\epsilon}$ fraction of constraints $\left(T_{i}, P_{i}\right)$ we have $\mathbb{E}_{x \sim \mu_{i}}\left[P_{i}(x)\right] \geq$ $1-\sqrt{\epsilon}$. In other words, $\mu_{i}$ has a $1-\sqrt{\epsilon}$ fraction of its mass on $P_{i}^{-1}(1)$.

Claim 4.2. Given a correlation matrix $\rho \in \mathbb{R}^{k \times k}$ of a distribution which is $(1-\sqrt{\epsilon})$-supported on $P^{-1}(1)$, there is a P-supported correlation matrix $\rho^{\prime}$ such that $\left|\rho(G)-\rho^{\prime}(G)\right| \leq \sqrt{\epsilon} 2^{m}$ for every $G$ on $m$ edges.

Thus, setting $\epsilon<\frac{\delta^{2}}{4 \cdot 2^{m}}$ we have that $\sum_{i} \gamma_{i} \rho\left(G_{i}\right)>\delta / 2$ for all correlation matrices of distributions which are $(1-\sqrt{\epsilon})$-supported on $P^{-1}(1)$.

Now the rounding algorithm is as in Figure 2 .

Now, fix the value of $t$ chosen in step 1 , and let $\alpha_{i, j}$ be the rounded value to the variable $x_{i, j}$ as in Lemma 4.1 and $\tilde{\alpha}_{i, j}$ be equal to $\alpha_{i, j}$ or its negation after the third step above. The assignment $\tilde{\alpha}$ is in $[-1,1]^{n \times k}$ but as the objective function is multilinear it can be greedily adjusted to an integral assignment in $\{-1,1\}^{n \times k}$ without decreasing the objective value, so it suffices to study $\tilde{\alpha}$. Let $S \subseteq[k]$ be some set of layers and $V: S \rightarrow[n]$ be any choice of variables from these layers. 
1. Pick $t \in[M]$ with probability $\left|\frac{\gamma_{t}}{\hat{P}\left(S_{t}\right)}\right| \cdot B^{\left|S_{t}\right|}$.

2. Using Lemma 4.1, round the variables in layers in $S_{t}$ using graph $G_{t}$. For every other layer, set all the variables in that layer to 0 .

3. If $\operatorname{sign}\left(\gamma_{t} \hat{P}\left(S_{t}\right)\right)=-1$, then select an odd sized subset $A$ of $S_{t}$ at random, else select an even sized subset $A$ of $S_{t}$ at random. Flip the sign of all variables in layers in $A$.

Fig. 2: Rounding algorithm for $\operatorname{MAx} \operatorname{PARTCSP}(P)$

Observe that for every $S \neq S_{t}$,

$$
\underset{\alpha}{\mathbb{E}}\left[\prod_{i \in S} \tilde{\alpha}_{i, V(i)}\right]=0 .
$$

To see this, note that if $S \nsubseteq S_{t}$, then the variables in layers $S \backslash S_{t}$ are set to 0 . On the other hand if $S \subset S_{t}$, then we flip the signs of a random set of layers of either odd or even size. As the distribution over which layers get flipped is $\left(\left|S_{t}\right|-1\right)$-wise independent, the layers of any $S \subset S_{t}$ get flipped completely uniformly.

On the other hand, by Lemma 4.1 and the way the signs are flipped in the third step, if $S=S_{t}$ we have

$$
\underset{\alpha}{\mathbb{E}}\left[\prod_{i \in S} \tilde{\alpha}_{i, V(i)}\right]=\operatorname{sign}\left(\gamma_{t} \hat{P}\left(S_{t}\right)\right) \cdot \frac{\rho\left(G_{t}\right)}{B^{\left|S_{t}\right|}} \pm \tau .
$$

Thus, taking the expectation of $\mathbb{E}_{\alpha}\left[\prod_{i \in S} \tilde{\alpha}_{i, V(i)}\right]$ over $t \in[M]$ chosen according to Step 1 , we have

$$
\begin{aligned}
\underset{t}{\mathbb{E}}\left[\underset{\alpha}{\mathbb{E}}\left[\prod_{i \in S} \tilde{\alpha}_{i, V(i)}\right]\right] & =\sum_{t: S_{t}=S}\left|\frac{\gamma_{t}}{\hat{P}\left(S_{t}\right)}\right| \cdot B^{\left|S_{t}\right|}\left(\operatorname{sign}\left(\gamma_{t} \hat{P}\left(S_{t}\right)\right) \cdot \frac{\rho\left(G_{t}\right)}{B^{\left|S_{t}\right|}} \pm \tau\right) \\
& =\sum_{t: S_{t}=S} \frac{\gamma_{t}}{\hat{P}\left(S_{t}\right)} \rho\left(G_{t}\right) \pm \tau
\end{aligned}
$$

Now we can analyze the probability that any specific constraint is satisfied. Let $\left(T_{i}, P_{i}\right)$ be a constraint involving one variable from each layer which is $(1-\sqrt{\epsilon})$-satisfied by the SDP solution. In other words, $\mathbb{E}_{x \sim \mu_{i}}\left[P_{i}(x)\right] \geq 1-\sqrt{\epsilon}$. Write $V:[k] \rightarrow[n]$ for the variables involved (i.e., $\left.T_{i}=\left\{\left(i^{\prime}, V\left(i^{\prime}\right)\right): i^{\prime} \in[k]\right\}\right)$ and write $P_{i}(x)=P\left(b_{1} x_{1, V(1)}, \ldots, b_{k} x_{k, V(k)}\right)$ for some signs $b_{1}, \ldots, b_{k} \in$ $\{-1,1\}$.

We also associate the domain of $P_{i}$ with $\{-1,1\}^{k}$ in the obvious way. As such, it is easy to verify that the Fourier coefficient $\hat{P}_{i}(S)$ for $S \subseteq[k]$ satisfies $\hat{P}_{i}(S)=\hat{P}(S) \chi_{S}(b)$. Furthermore, let $\tilde{\mu}_{i}$ be the distribution over $\{-1,1\}^{k}$ obtained by sampling from $\mu_{i}$ and performing coordinatewise multiplication by $b$, and let $\rho$ (resp. $\tilde{\rho}$ ) denote the correlation matrix of $\mu_{i}$ (resp. $\tilde{\mu}_{i}$ ). Then, for any graph $G$ we have

$$
\rho(G)=\prod_{\left(a, a^{\prime}\right) \in E} \rho_{a, a^{\prime}}=\prod_{\left(a, a^{\prime}\right) \in E} \tilde{\rho}_{a, a^{\prime}} \chi_{\left\{a, a^{\prime}\right\}}(b)=\tilde{\rho}(G) \cdot \chi_{\operatorname{Odd}(G)}(b)
$$

where $\operatorname{Odd}(G)$ denotes the set of odd-degree vertices of $G$. In particular for $G=G_{t}$ all vertices have odd degree so $\operatorname{Odd}\left(G_{t}\right)=S_{t}$. Using this and noting that $\tilde{\mu}_{i}$ is $(1-\sqrt{\epsilon})$-supported on satisfying 
assignments of $P$ we see that

$$
\sum_{t} \gamma_{t} \rho\left(G_{t}\right) \chi_{S_{t}}(b)=\sum_{t} \gamma_{t} \tilde{\rho}\left(G_{t}\right)>\delta / 2
$$

We then have the following, where the expectation below is taken over all the random choices of the algorithm (including that of $t \in[M]$ ).

$$
\begin{aligned}
\mathbb{E}\left[P_{i}\left(\tilde{\alpha}_{1, V(1)}, \cdots, \tilde{\alpha}_{k, V(k)}\right)\right] & =\hat{P}_{i}(\emptyset)+\sum_{\substack{\emptyset \neq S \subseteq[k] \\
\hat{P}_{i}(S) \neq 0}} \hat{P}_{i}(S) \mathbb{E}\left[\prod_{i \in S} \tilde{\alpha}_{i, V(i)}\right] \\
& =\hat{P}(\emptyset)+\sum_{\substack{\emptyset \neq S \subseteq[k] \\
\hat{P}(S) \neq 0}} \hat{P}(S) \chi_{S}(b) \sum_{t: S_{t}=S} \frac{\gamma_{t}}{\hat{P}\left(S_{t}\right)} \rho\left(G_{t}\right) \pm \tau \\
& =\hat{P}(\emptyset)+\sum_{t=1}^{M} \gamma_{t} \rho\left(G_{t}\right) \chi_{S_{t}}(b) \pm \tau M>\hat{P}(\emptyset)+\frac{\delta}{4} .
\end{aligned}
$$

Thus the total fraction of constraints satisfied by the algorithm is in expectation at least $(1-$ $\sqrt{\epsilon})(\hat{P}(\emptyset)+\delta / 4)$ which is at least $\hat{P}(\emptyset)+\delta / 8$ assuming $\epsilon<(\delta / 8)^{2}$.

In other words, the algorithm finds a $(\hat{P}(\emptyset)+\delta / 8)$-approximate solution on all instances with value at least $1-\epsilon$. Combining this with a random assignment gives an approximation better than $\hat{P}(\emptyset)$ for any instance, and concludes the proof of approximability of MAX PARTCSP $(P)$.

\section{Hardness}

In this section we show that any $P$ which satisfies the condition of Theorem 1.3 is approximation resistant, assuming the UGC. As usual, we prove hardness by designing an appropriate dictatorship test, which is given in Section 5.1, followed by the (standard) hardness reduction in Section 5.2.

\subsection{Dictatorship Test}

Theorem 5.1. Let $P$ satisfy the condition of Theorem 1.3. Then for every $k$ and $\epsilon>0$ there exists a $\delta>0$ such that the following holds for all $n$.

There is a randomized algorithm $\mathcal{T}$ which, given oracle access to $k$ odd functions $f_{1}, \ldots, f_{k}$ : $\{-1,1\}^{n} \rightarrow[-1,1]$, produces $k$ queries $X_{1}, \ldots, X_{k} \in\{-1,1\}^{n}$ such that

(Yes) If $f_{1}(x)=f_{2}(x)=\ldots=f_{k}(x)=x_{i}$ are the same dictator function, then $\mathbb{E}\left[P\left(f_{1}\left(X_{1}\right), \ldots, f_{k}\left(X_{k}\right)\right)\right] \geq$ $1-\epsilon$.

(No) If all $f_{i}$ 's have $\operatorname{Inf}_{j}^{\leq 1 / \delta}\left(f_{i}\right) \leq \delta$ for all $j \in[n]$ then $\mathbb{E}\left[P\left(f_{1}\left(X_{1}\right), \ldots, f_{k}\left(X_{k}\right)\right)\right] \leq \hat{P}(\emptyset)+\epsilon$

Let $m=k \cdot n$ and let $\Lambda$ be a distribution over $P$-supported correlation matrices which is $m$ vanishing on $P$. Let $U_{k}$ be the uniform distribution over $\{-1,1\}^{k}$. The tester $\mathcal{T}$ is described in Figure 3

That the completeness is $1-\epsilon$ follows immediately from $\operatorname{supp}(\eta) \subseteq P^{-1}(1)$.

Let us then analyze the soundness. The acceptance probability can be written as

$$
\begin{aligned}
\operatorname{Pr}[\mathcal{T} \text { accepts }] & =\underset{\rho \sim \Lambda}{\mathbb{E}}\left[\underset{X \sim \mu}{\mathbb{E}}\left[P\left(f_{1}\left(X_{1}\right), \ldots, f_{k}\left(X_{k}\right)\right)\right]\right] \\
& =\hat{P}(\emptyset)+\underset{\rho}{\mathbb{E}}\left[\sum_{S \neq \emptyset} \hat{P}(S) \underset{X}{\mathbb{E}}\left[\prod_{i \in S} f_{i}\left(X_{i}\right)\right] .\right.
\end{aligned}
$$


Input: functions $f_{1}, \ldots, f_{k}:\{-1,1\}^{n} \rightarrow[-1,1]$

Output: accept/reject

1. Pick a random $\rho \sim \Lambda$.

2. Let $\eta$ be an unbiased $P$-supported distribution with correlation matrix $\rho$ (if there are many such $\eta$ pick an arbitrary one in a deterministic fashion).

3. Let $\mu=(1-\epsilon) \eta+\epsilon U_{k}$.

4. Pick a random $k \times n$ matrix $X$ where each column is sampled independently according to $\mu$.

5. Accept with probability $P\left(f_{1}\left(X_{1}\right), \ldots, f_{k}\left(X_{k}\right)\right)$.

Fig. 3: Dictatorship Test

Let $\rho^{\prime}$ be the correlation matrix of $\mu$. Note that $\rho^{\prime}=(1-\epsilon) \rho+\epsilon I$. Fix the value of $\rho$ for the moment, and let $G$ be a random $k \times n$ matrix of standard Gaussians with the same covariances as $X$ (i.e., the columns are independent and in the $j^{\prime}$ th column we have $\mathbb{E}\left[G_{i, j} G_{i^{\prime}, j}\right]=\rho_{i, i^{\prime}}^{\prime}=(1-\epsilon) \rho_{i, i^{\prime}}$ for $i \neq i^{\prime}$ ).

Next, set $\delta$ small enough so that Theorem 2.6 gives that if $\operatorname{Inf}_{j}^{\leq 1 / \delta}\left(f_{i}\right) \leq \delta$ for all $i \in[k], j \in[n]$ then

$$
\left|\underset{X}{\mathbb{E}}\left[\prod_{i \in S} f_{i}\left(X_{i}\right)\right]-\underset{G}{\mathbb{E}}\left[\prod_{i \in S} f_{i}^{\leq 1 / \delta}\left(G_{i}\right)\right]\right| \leq \epsilon / 2^{k}
$$

for all $S$. Define $f_{i}^{\prime}=f \leq 1 / \delta$. We need to understand expressions of the form $\mathbb{E}_{G}\left[\prod_{i \in S} f_{i}^{\prime}\left(G_{i}\right)\right]$ for $S \subseteq[k]$. Expanding $f_{i}^{\prime}=\sum_{T \subseteq[n]} \hat{f}^{\prime}(T) \chi_{T}$ and applying Lemma 2.7, we obtain

$$
\begin{aligned}
\underset{G}{\mathbb{E}}\left[\prod_{i \in S} f_{i}^{\prime}\left(G_{i}\right)\right] & =\sum_{\left\{T_{i}\right\}_{i \in S}} \prod_{i \in S} \hat{f}_{i}^{\prime}\left(T_{i}\right) \prod_{j=1}^{n} \mathbb{E}\left[\prod_{i: j \in T_{i}} G_{i j}\right] \\
& =\sum_{\left\{T_{i}\right\}_{i \in S}} \prod_{i \in S} \hat{f}_{i}^{\prime}\left(T_{i}\right) \prod_{j=1}^{n} \sum_{M_{j} \in \mathcal{M}\left(\left\{i: j \in T_{i}\right\}\right)} \prod_{i, i^{\prime} \in M_{j}} \rho_{i, i^{\prime}}^{\prime}
\end{aligned}
$$

where we write $\mathcal{M}(S)$ for the set of perfect matchings on the complete graph with vertex set $S$.

For a choice $\underline{\mathrm{T}}=\left\{T_{i}\right\}_{i \in S}$ of $T_{i}$ 's, let $c(\underline{\mathrm{T}})=\prod_{i \in S} \hat{f}_{i}^{\prime}\left(T_{i}\right)$. Further, for a choice of matchings $\underline{\mathrm{M}}=\left(M_{1}, \ldots, M_{n}\right)$ let $H(\underline{\mathrm{M}})$ denote the multigraph being the union of $M_{1}, \ldots, M_{n}$. With a slight abuse of notation, write $\mathcal{M}(\underline{\mathrm{T}})$ for the set of $\underline{\mathrm{M}}$ 's for a given $\underline{\mathrm{T}}$; i.e., $\mathcal{M}(\underline{\mathrm{T}})=\left\{\left(M_{1}, \ldots, M_{n}\right): M_{j} \in\right.$ $\mathcal{M}\left(\left\{i: j \in T_{i}\right\}\right)$. With all this cumbersome notation in place, the equation above simplifies to

$$
\text { (44) }=\sum_{\underline{\mathrm{T}}} \sum_{\underline{\mathrm{M}} \in \mathcal{M}(\underline{\mathrm{T}})} c(\underline{\mathrm{T}}) \rho^{\prime}(H(\underline{\mathrm{M}})),
$$

where $\underline{T}$ ranges over all $\left\{T_{i} \subseteq[n]\right\}_{i \in S}$. Note that since $f_{i}$ (and therefore also $f_{i}^{\prime}$ ) is odd, we can restrict the sum to $\underline{\mathrm{T}}$ such that each $\left|T_{i}\right|$ is odd, implying that $H(\underline{\mathrm{M}})$ is always odd degree. 
Plugging (15) into (3), we have

$$
\left|\underset{X}{\mathbb{E}}\left[\prod_{i \in S} f_{i}\left(X_{i}\right)\right]-\sum_{\underline{\mathrm{T}}} \sum_{\underline{\mathrm{M}} \in \mathcal{M}(\underline{\mathrm{T}})} c(\underline{\mathrm{T}}) \rho^{\prime}(H(\underline{\mathrm{M}}))\right| \leq \epsilon / 2^{k}
$$

Finally, plugging this into (2) and using the identity $\rho^{\prime}=(1-\epsilon) \rho+\epsilon I$ yields

$$
\begin{aligned}
\operatorname{Pr}[\mathcal{T} \text { accepts }] & \leq \hat{P}(\emptyset)+\underset{\rho}{\mathbb{E}}\left[\sum_{S \neq \emptyset}\left(\hat{P}(S) \sum_{\underline{\mathrm{T}}} \sum_{\underline{\mathrm{M}} \in \mathcal{M}(\underline{\mathrm{T}})} c(\underline{\mathrm{T}}) \rho^{\prime}(H(\underline{\mathrm{M}}))+\epsilon / 2^{k}\right)\right] \\
& \leq \hat{P}(\emptyset)+\epsilon+\sum_{S \neq \emptyset} \hat{P}(S) \sum_{\underline{\mathrm{T}}} \sum_{\underline{\mathrm{M}} \in \mathcal{M}(\underline{\mathrm{T}})} c(\underline{\mathrm{T}})(1-\epsilon)^{|E(H(\underline{\mathrm{M}}))|} \underset{\rho}{\mathbb{E}}[\rho(H(\underline{\mathrm{M}}))] \\
& =\hat{P}(\emptyset)+\epsilon,
\end{aligned}
$$

where the last equality follows by the $m$-vanishing property of $\Lambda$ : $H(\underline{\mathrm{M}})$ has at most $n|S| / 2<n k=$ $m$ edges, and so for each $S$ either $\hat{P}(S)=0$ or $\mathbb{E}_{\rho}[\rho(H(\underline{\mathrm{M}}))]=\Lambda(H(\underline{\mathrm{M}}))=0$.

\subsection{Hardness Reduction}

Given the dictatorship test as in Theorem 5.1, a UGC-based hardness reduction can be designed in a standard manner. Some care needs to be taken however to ensure that the CSP instance produced by the reduction is $k$-partite. As is standard, we present the reduction as a Probabilistically Checkable Proof (PCP) for NP whose acceptance predicate matches the predicate $P$, has completeness $1-o(1)$ and soundness $\frac{\left|P^{-1}(1)\right|}{2^{k}}+o(1)$.

The PCP is based on the conjectured NP-hard instance $\Lambda=(U, V, E, \Pi,[L])$ of Unique Games as in Definition 2.1. Let $L$ and $\gamma$ be as in Conjecture 2.3. The PCP proof consists of $k$ layers where the bits in the $i$ 'th layer correspond to $V_{i} \times\{-1,1\}^{L}$ and $V_{i}$ is a copy of the "right hand side" $V$ of the UG instance. For any $v_{i} \in V_{i}(=V)$, the set of bits $\left\{v_{i}\right\} \times\{-1,1\}^{L}$ correspond to the bits of the long code of the label of $v_{i}$. In a "correct" proof, the assignment to these bits corresponds to a dictatorship function $f(x)=x_{j}$ where $j \in[L]$ is the intended label of $v_{i}$.

For a function $g:\{-1,1\}^{L} \rightarrow\{-1,1\}$ and a permutation $\pi:[L] \rightarrow[L]$, let $g \circ \pi^{-1}:\{-1,1\}^{L} \rightarrow$ $\{-1,1\}$ denote the function defined as $g \circ \pi^{-1}(x)=g\left(x_{\pi^{-1}(1)}, \ldots, x_{\pi^{-1}(L)}\right)$. The PCP verifier proceeds as in Figure 4.

1. Pick a random vertex $u \in U$.

2. Pick $k$ random neighbors of $u$, namely $v_{1}, \ldots, v_{k} \in V$.

3. Let $g_{1}, \ldots, g_{k}$ be the functions (supposed long codes) for $v_{1} \in V_{1}, \ldots, v_{k} \in V_{k}$ respectively.

4. Let $f_{1}, \ldots, f_{k}$ be the permuted versions of $g_{1}, \ldots, g_{k}$ respectively, i.e., $f_{i}=g_{i} \circ \pi_{i}^{-1}$, $\pi_{i}=\pi_{e_{i}=\left(u, v_{i}\right)}$ for $1 \leq i \leq k$.

5. Run the dictatorship test as in Theorem 5.1 on $\left(f_{1}, \ldots, f_{k}\right)$.

Fig. 4: PCP Verifier 


\subsubsection{Completeness}

Let $\ell: U \cup V \rightarrow[L]$ be a labeling to the UG instance that satisfies $1-\gamma$ fraction of its edges. For every $v_{i} \in V_{i}(=V)$, let $g_{i}$ be the long code of $\ell\left(v_{i}\right)$, i.e. $g_{i}(x)=x_{\ell\left(v_{i}\right)}$. With probability at least $1-k \gamma$, all $k$ edges $\left(u, v_{1}\right), \ldots,\left(u, v_{k}\right)$ are satisfied by the labeling and whenever this holds, the dictatorship test accepts with probability $1-\epsilon$. The latter conclusion follows by observing that if $\pi_{i}(\ell(u))=\ell\left(v_{i}\right)$ for every $1 \leq i \leq k$, then in the PCP test above,

$$
f_{i}(x)=g_{i} \circ \pi_{i}^{-1}(x)=g_{i}\left(x_{\pi_{i}^{-1}(1)}, \ldots, x_{\pi_{i}^{-1}(L)}\right)=x_{\pi_{i}^{-1}\left(\ell\left(v_{i}\right)\right)}=x_{\ell(u)},
$$

and hence $f_{1}, \ldots, f_{k}$ are identical dictatorship functions.

\subsubsection{Soundness}

Assume that the soundness of the UG instance is at most $\gamma$ which is chosen to be sufficiently small beforehand. Fix any layer $i$ in the PCP proof and let $g_{i, v}$ be the supposed long code corresponding to the vertex $v$ (in the $i$ 'th layer). For any $u \in U$, define the function $f_{i, u}$ which is the average of functions over the neighbors of $u$ after appropriate permutation:

$$
f_{i, u}(x)=\underset{v:(u, v) \in E}{\mathbb{E}}\left[g_{i, v} \circ \pi_{(u, v)}^{-1}(x)\right] .
$$

Note that $f_{i, u}$ are $[-1,1]$-valued. By a standard argument, we may assume that for all but $\sqrt{\gamma}$ fraction of $u \in U$, the function $f_{i, u}$ has no coordinate that has degree $1 / \delta$ influence $\delta$ (referred to as a low-influence function for brevity).

Otherwise, suppose that for $\sqrt{\gamma}$ fraction of $u, f_{i, u}$ has a coordinate that has degree $1 / \delta$ influence $\delta$. For brevity, call any such coordinate simply as an influential coordinate. The set of all influential coordinates has size bounded by $1 / \delta^{2}$. Assign this bounded set as the set of candidate labels for $u$. For any influential coordinate $j \in[L]$, since $f_{i, u}$ is an average of $g_{i, v} \circ \pi_{(u, v)}^{-1}$ over neighbors of $u$, by an averaging argument, for at least $\delta / 2$ fraction of the neighbors, $\pi_{(u, v)}(j)$ is influential for $g_{i, v}$. All influential coordinates of $g_{i, v}$ are assigned as the candidate labels for $v$. Now define a (randomized) labeling that selects one label at random from the candidate set of each vertex. The argument sketched implies that this labeling satisfies $\sqrt{\gamma} \cdot \delta / 2 \cdot \delta^{4}$ fraction of the UG edges. This is a contradiction if the soundness $\gamma$ was chosen to be sufficiently small to begin with.

Hence except with probability $k \sqrt{\gamma}$, the PCP verifier chooses $u \in U$ such that the $k$ functions $f_{i, u}$, one in each layer, are all low influence functions. Whenever this holds, the analysis of the dictatorship test implies that the verifier accepts with probability at most $\frac{\left|P^{-1}(1)\right|}{2^{k}}+\epsilon$. One only needs to observe that for a fixed $u$, the verifier picks its random neighbor in each layer and hence running the test on these random neighbors (one in each layer) has the same effect as running the test on the (possibly non-boolean) averaged functions (again, one in each layer). Formally, fixing $u$,

$$
\begin{aligned}
\underset{v_{1}, \ldots, v_{k}}{\mathbb{E}}\left[\underset{\mathcal{T}}{\mathbb{E}}\left[P\left(g_{1, v_{1}} \circ \pi_{1}^{-1}\left(X_{1}\right), \ldots, g_{k, v_{k}} \circ \pi_{k}^{-1}\left(X_{k}\right)\right)\right]\right] \\
=\underset{\mathcal{T}}{\mathbb{E}}\left[P\left(\underset{v_{1}}{\mathbb{E}}\left[g_{1, v_{1}} \circ \pi_{1}^{-1}\left(X_{1}\right)\right], \ldots, \underset{v_{k}}{\mathbb{E}}\left[g_{k, v_{k}} \circ \pi_{k}^{-1}\left(X_{k}\right)\right]\right)\right] \\
=\underset{\mathcal{T}}{\mathbb{E}}\left[P\left(f_{1, u}\left(X_{1}\right), \ldots, f_{k, u}\left(X_{k}\right)\right)\right] .
\end{aligned}
$$




\section{Acknowledgements}

We are grateful to Johan Håstad for many insightful discussions throughout this work, and to the anonymous referees for their helpful suggestions.

\section{References}

[AH11] P. Austrin and J. Håstad. Randomly supported independence and resistance. SIAM Journal on Computing, 40:1-27, 2011.

[AH12] P. Austrin and J. Håstad. On the Usefulness of Predicates. In IEEE Conference on Computational Complexity (CCC), 2012.

[AM09] P. Austrin and E. Mossel. Approximation resistant predicates from pairwise independence. Computational Complexity, 18:249-271, 2009.

[EH08] L. Engebretsen and J. Holmerin. More efficient queries in PCPs for NP and improved approximation hardness of maximum CSP. Random Structures and Algorithms, 33:497$514,2008$.

[GLST98] V. Guruswami, D. Lewin, M. Sudan, and L. Trevisan. A tight characterization of NP with 3 query PCPs. In Proceedings of 39th Annual IEEE Symposium on Foundations of Computer Science, pages 8-17, Palo Alto, 1998. IEEE.

[GW95] M. Goemans and D. Williamson. Improved approximation algorithms for maximum cut and satisfiability problems using semidefinite programming. Journal of the ACM, 42:1115-1145, 1995.

[Hås01] J. Håstad. Some optimal inapproximability results. Journal of ACM, 48:798-859, 2001.

[Has05] G. Hast. Beating a random assignment. KTH, Stockholm, 2005. Ph.D Thesis.

[Kho02] S. Khot. On the power of unique 2-prover 1-round games. In Proceedings of 34th ACM Symposium on Theory of Computating, pages 767-775, 2002.

[MOO10] E. Mossel, R. O'Donnell, and K. Oleszkiewicz. Noise stability of functions with low influences: invariance and optimality. Annals of Mathematics, 171(1):295-341, 2010.

[Mos10] E. Mossel. Gaussian bounds for noise correlation of functions. GAFA, 19:1713-1756, 2010.

[Rag08] P. Raghavendra. Optimal Algorithms and Inapproximability Results For Every CSP? In ACM Symposium on Theory of Computing (STOC), 2008.

[RS09] P. Raghavendra and D. Steurer. How to Round Any CSP. In IEEE Symposium on Foundations of Computer Science (FOCS), pages 586-594, 2009.

[ST00] A. Samorodnitsky and L. Trevisan. A PCP characterization of NP with optimal amortized query complexity. In Proceedings of the 32nd Annual ACM Symposium on Theory of Computing, pages 191-199, 2000.

[Tar51] A. Tarski. A decision method for elementary algebra and geometry. Bulletin of the American Mathematical Society, 59, 1951. 
[Zwi98] U. Zwick. Approximation algorithms for constraint satisfaction problems involving at most three variables per constraint. In Proceedings 9th Annual ACM-SIAM Symposium on Discrete Algorithms, pages 201-210. ACM, 1998. 